\title{
FIR-based Classifiers for Animal Behavior Classification
}

\author{
Majid M. Beigi and Andreas Zell
}

\begin{abstract}
In this paper, we implement a new method for classification of biological signals in general, and use it in the animal behavior classification as an example. The forced swimming test of rats or mice is a frequently used behavioral test to evaluate the efficacy of drugs in rats or mice. Frequently used features for that evaluation are obtained through observing three states: immobility, struggling/climbing and swimming in activity profiles. We consider that those activity profiles (signals) inherently contain undesired and interference noise that should be removed before feature extraction and classification. We use a Finite Impulse Response (FIR) filter to filter out that additive noise from the activity profile. The parameters of the FIR filter are obtained via maximizing the accuracy of a classifier that tries to make a discrimination between two classes of the activity profiles (e.g. drug vs. control). We use the kernel Fisher discriminant criterion as a criterion for the discrimination, the DIviding RECTangles (DIRECT) search method for solving the optimization problem and Support Vector Machines (SVMs) for the classification task. We show that Autoregressive (AR) coefficients are suitable features for the extraction of the dynamic behavior of rats and also the classification of activity profiles. Our proposed behavior classification method provides a reliable discrimination of different classes of antidepressant drugs (imipramine and desipramine) administered to rats versus a vehicle-treated group.
\end{abstract}

\section{INTRODUCTION}

The Forced Swimming Test (FST) is a behavioral test used frequently to evaluate the potential efficacy of drugs affecting the central nervous system (CNS) in rats or mice [3]. In this experiment, rats are exposed to a 15 -min pretest swim period and followed the next day by a 5 -min test swim. Immersion of rodents in water for an extended period of time produces a characteristic behavior called immobility, in which the rat makes only those movements necessary to keep its head above water. When antidepressant drugs are administered between the pretest and test periods, usually three times within $24 \mathrm{hr}$, the behavioral immobility is selectively decreased [4]. Depending on the type of drug, rats show a mixed behavior of activities such as immobility, struggling/climbing (the rat tries to escape from the water) and swimming. Researchers have tried to conclude the effect of drugs from the above three states (immobile, struggling and swimming) [25]. Typically, tricyclic antidepressants and drugs with selective effects on noradrenergic transmission increase struggling/climbing behavior, while selective serotonin reuptake inhibitors increase swimming behavior versus the control group [7], [5], [6].

M. M. Beigi is $\mathrm{PhD}$. student at the Department of Computer Science, University of Tuebingen, 72076 Tuebingen, Germany. majid.beigi@uni-tuebingen.de

A. Zell is Professor at the Department of Computer Science, University of Tuebingen, 72076 Tuebingen, Germany. Andreas.Zell@uni-tuebingen.de

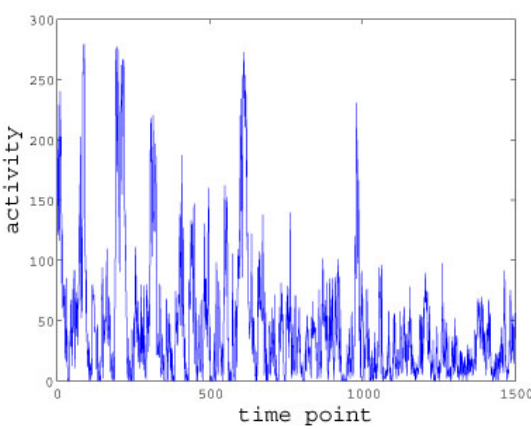

Tylose

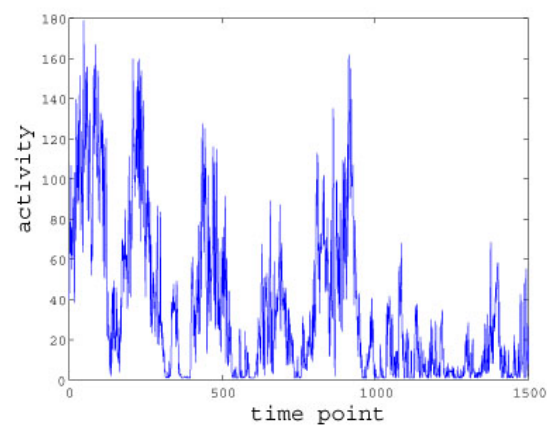

Desipramine $30 \mathrm{mg}$

Fig. 1. FST test as a behavioral test. The successive images of rat movement are converted to activity profile signals. a) activity profile (arbitrary unit) for tylose as control and b) Desipramine 30mg as antidepressant.

Fig. 1 shows examples of activity profiles, which are gained from successive images of rat movement in FST test. Considering predefined thresholds for the immobility, struggling and swimming states, and depending on the amplitude of the activity profile, the average period of time in each state can be measured. By comparing those parameters for the animals treated with an antidepressant against the control group, which was treated with the vehicle, it can be observed, for example, whether the swimming behavior of rats with an antidepressant drug has increased.

In an automated classification method, we aim to classify animals treated with known antidepressants and the control group. However, our experiments show that the response of the rat to drugs is too complex to only consider those states as indicator of the drug efficacy. The detection of the behavior of rats depends on recognizing changes in some characteristics of movement and we are interested in features which represent also the dynamic behavior of rats.

An important issue in the automated classification is the presence of noise in activity profiles. We consider the activity profile, $x(n)$, as the total of the inherent response of a rat to a drug at a certain dose, $s(n)$, and the undesired and 
interference noise, $N(n)$, so we have:

$$
x(n)=s(n)+N(n)
$$

$N(n)$ is the undesired part, which affects the accuracy of classifier. The presence of this noise can be due to the experimental setup, the difference between the physiology behavior of rats and so on. One method to remove this noise is to use a suitable filter based on its frequency content. But the frequency content of this noise is unknown. To resolve this problem, we use the fact that when a suitable filter is added to a classifier, it should increase the signal to noise ratio and so the accuracy of the classifier. For this, we use a general model of filters known as FIR (Finite Impulse Response):

$$
\mathrm{W}_{n}=\left[w_{n}(0), w_{n}(1), \ldots, w_{n}(p-1)\right]^{T},
$$

and the output of that filter is the estimation of $s(n)$ :

$$
\hat{s}(n)=\mathrm{W}_{n}^{T} x(n)
$$

where the coefficients of the FIR filter are obtained via optimizing a criterion showing the accuracy of a classifier which tries to classify two different classes of signals.

Recently, Kernel Fisher Discriminant Analysis (KFDA) has been used for classification and also for optimal kernel selection [1], [2], [8]. In this study we use the Fisher discriminant criterion in the kernel space as a criterion for the accuracy of the classifier and try to find the optimal coefficient of the FIR filter that maximizes that criterion. To solve the optimization problem, we use the DIRECT search method as an algorithm for global nonlinear optimization

We also consider the activity profiles as outputs of a black box system and select a suitable model which extracts the dynamic behavior of those activity profiles. One possible representation of the local dynamic behavior in the profile activity is using an autoregressive (AR) model, in which future values are predicted from a combination of previous sample values. After the FIR filter, we use AR coefficients as features for the extraction of the dynamic behavior and also the classification of activity profiles.

In the following sections we implement the FIR based classifier in detail and then we explain the experimental setup and results of classification. At last, we show our conclusions.

\section{ALGORITHMS}

\section{A. FIR filter based classifier}

We consider the problem of classification of signals that contain additive unknown or undesired parts interference (noise). If we can not find suitable features representing the desired part of signals or if we can not deduce the interference signal from the original signal, the classifier may face overfitting and so a decrease of the accuracy.

FIR filters are designed to filter out the undesired part from the signal based on its frequency specification. Because of its stability and simplicity in implementation, they are

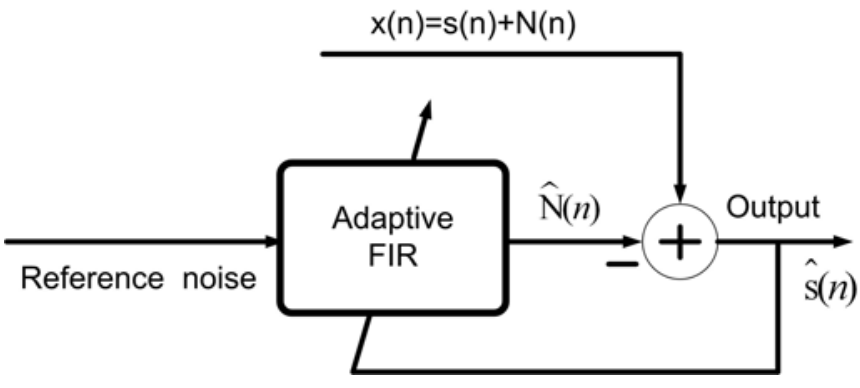

Fig. 2. Adaptive filter for noise cancellation

used frequently in different applications. However, in some applications (such as FST classification), we do not have the frequency specification of the noise, needed to design the FIR filter.

Fig. 2 shows the block diagram of an adaptive filter which is used for noise (interference) cancellation. As we know if the reference noise is uncorrelated to the signal $s(n)$, the output of the FIR filter is an estimation of the interference $(\hat{N}(n))$ and the filter readjusts itself continuously to minimize the error between $N$ and $\hat{N}$. The coefficients of the FIR filter can be obtained through the Least Mean Square (LMS) algorithm. But in our task the stationary and uncorrelatedness conditions for $s$ and $N$ are not met and we can not estimate $s(n)$ using the adaptive filter. To cope with the problem, we suggest a new solution.

We propose a FIR filter based classifier, in which the FIR filter tries to remove undesired parts by getting feedback from the accuracy of a following classifier. Fig. 3 shows our proposed solution. Consider $x_{1}(n)=s_{1}(n)+N_{1}(n)$ and $x_{2}(n)=s_{2}(n)+N_{2}(n)$ as two signals that contain undesired parts $N_{1}(n)$ and $N_{2}(n)$. Our aim is to estimate $s_{1}(n)$ and $s_{2}(n)$ as outputs of the added FIR filter. If we consider the $l$-length coefficient of the FIR filter as:

$$
\mathrm{W}_{n}=\left[w_{n}(0), w_{n}(1), \ldots, w_{n}(l-1)\right]^{T}
$$

we have:

$$
\hat{s}_{1}(n)=\mathrm{W}_{n}^{T} x_{1}(n), \hat{s}_{2}(n)=\mathrm{W}_{n}^{T} x_{2}(n)
$$

The classifier should discriminate $\hat{s}_{1}(n)$ from $\hat{s}_{2}(n)$. The coefficients of the FIR filter that increases the accuracy of the classifier also increases the signal to noise ratio. Then, the task is to find optimum values of the coefficients that maximize a criterion showing the accuracy of the classifier. We select the Kernel Fisher discriminant criterion as a suitable objective for our optimization problem.

\section{B. Kernel Fisher discriminant based optimal FIR filter}

We want to find the coefficients of the FIR filter that maximizes the performance of the classifier. Here, we select the Fisher discriminant criterion in the kernel space as a criterion for the performance of the classifier. The kernel Fisher discriminant analysis (KFDA) [1] is a non-linear extension of the linear Fisher discriminant analysis. It finds 


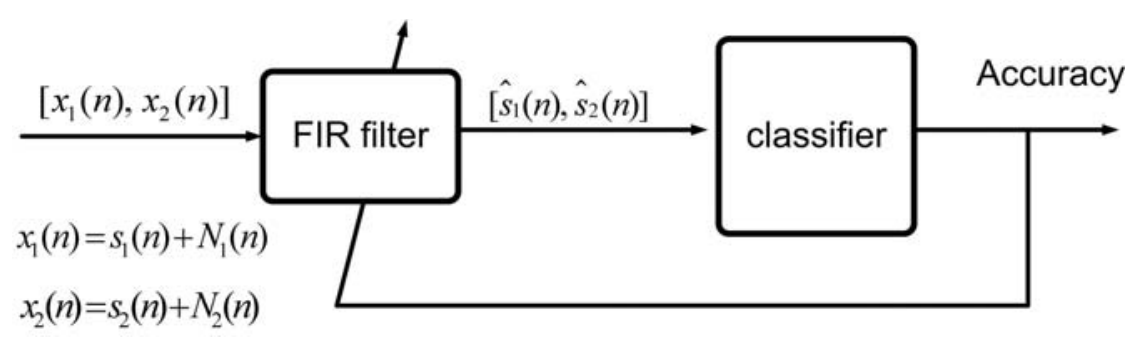

Fig. 3. Optimal FIR filter based classifier. The coefficients of FIR filters are gained via optimization of a criterion showing the accuracy of the classifier

the direction in a feature space, defined implicitly by a kernel, onto which the projections of positive and negative classes are well separated in terms of the Fisher discriminant ratio. The more two classes are separated, the higher the performance of the classifier is. Then, we make a relation between that criteria and the FIR filter coefficients and find the coefficients that maximizes that criterion. The kernel Fisher discriminant based optimal FIR filter tries to find the coefficients of the FIR filter that results in a vector $v$ in feature space, on which projections of points give the maximum separation of the mean scaled in the feature space and the minimum variance in that direction (Fig. 4).

The KFDA first maps the data via a non-linear mapping $\phi$ into the high dimensional feature space $\mathscr{F}$ and then optimizes the Fisher criterion. Given a map $\phi: u \rightarrow \phi(u) \in \mathscr{F}$, the aim is to find a direction $v=\sum_{i=1}^{n} \alpha_{i} \phi\left(u_{i}\right)$ in the feature space $\mathscr{F}$ given by weights $\alpha=\left[\alpha_{1}, \ldots, \alpha_{n}\right]$, that maximizes the separation of the mean scaled in the feature space and minimizes the variance in that direction (KFD criterion). Considering the kernel matrix $K$ :

$$
K_{i, j}=k\left(x_{i}, x_{j}\right)=<\phi\left(x_{i}\right), \phi\left(x_{j}\right)>
$$

For the direction $v$, the Fisher criterion will be in the form of ([1]):

$$
J(\alpha)=\frac{\alpha^{T} M \alpha}{\alpha^{T}(N+\lambda I) \alpha}
$$

The parameter $\lambda$ is a regulation factor and $M$ and $N$ (defined in [1]) are gained in terms of the kernel matrix $K$ :

$$
M=\left(\mu_{+}-\mu_{-}\right)\left(\mu_{+}-\mu_{-}\right)^{T}
$$

where $\mu_{+}=\frac{1}{n_{+}} \sum_{x \in \chi_{+}} K_{x}$, and $\mu_{-}=\frac{1}{n_{-}} \sum_{x \in \chi_{-}}$are scaled mean in the feature space, and:

$$
\begin{gathered}
N=K D K^{T} \\
\text { where } D=\left[\begin{array}{cc}
I_{n_{+}}-\frac{1}{n_{+}} 1_{n_{+}} 1_{n_{+}}^{T} & 0 \\
0 & I_{n_{-}}-\frac{1}{n_{-}} 1_{n_{-}} 1_{n_{-}}^{T}
\end{array}\right]_{n \times n}
\end{gathered}
$$

in which $1_{n}$ and $I_{n}$ denote the vector of all ones and the identity operator in $\mathbb{R}^{d}$, respectively.

It can be shown [2], the parameter $\alpha$ that maximizes Eq. 2 is obtained via:

$$
\alpha_{\max }=(N+\lambda I)^{-1}\left(\mu_{+}-\mu_{-}\right)=\left(K D K^{T}+\lambda I\right)^{-1} K y
$$

where:

$$
y=\left[\begin{array}{c}
\left(1 / n_{+}\right) 1_{n_{+}} \\
\left(-1 / n_{-}\right) 1_{n_{-}}
\end{array}\right]_{n \times 1}
$$

which results in:

$$
J_{\max }(K)=\alpha_{\max }^{T} K y=y^{T} K\left(K D^{T} K+\lambda I\right)^{-1} K y
$$

The above equation (Eq. 3 ) shows that the KFD criterion can be represented in terms of a kernel matrix.

The next step is to represent the kernel matrix in terms of the FIR coefficients. In Fig. 4, if we consider the $l$-length coefficient of the FIR filter as:

$$
\mathrm{W}_{n}=\left[w_{n}(0), w_{n}(1), \ldots, w_{n}(l-1)\right]^{T}
$$

we have:

$$
\hat{s}_{1}(n)=\mathrm{W}_{n}^{T} x_{1}(n), \hat{s}_{2}(n)=\mathrm{W}_{n}^{T} x_{2}(n)
$$

We extract the features $u_{1}$ and $u_{2}$ from $\hat{s}_{1}(n)$ and $\hat{s}_{1}(n)$ resp. and then:

$$
k\left(u_{1}, u_{2}\right)=<\phi\left(u_{1}\right), \phi\left(u_{2}\right)>
$$

From the above equation and equation (3) we can say that when the coefficients of the FIR filter vary, the KFD criterion varies, too. However, the task is to find the optimum coefficients of the FIR filter that maximizes the KFD criterion, i.e., maximizes the signal to interference ratio. For this, we use a method of global optimization, by which we can search for the optimum global value of the FIR coefficients in a given range.

\section{Direct search}

The DIRECT algorithm as an effective pattern search method was proposed by Jones et al. [17] for bound constrained global optimization. It deals with problems of the form

$$
\begin{array}{ll}
\min _{x} & f(x) \\
\text { s.t. } & x_{L} \leq x \leq x_{U},
\end{array}
$$

where $f \in \mathbb{R}$ and $x, x_{L}, x_{U} \in \mathbb{R}^{n}$.

The DIRECT algorithm is one of a class of deterministic direct search algorithms that does not require gradients. If the objective function $f$ is continuous or at least continuous 


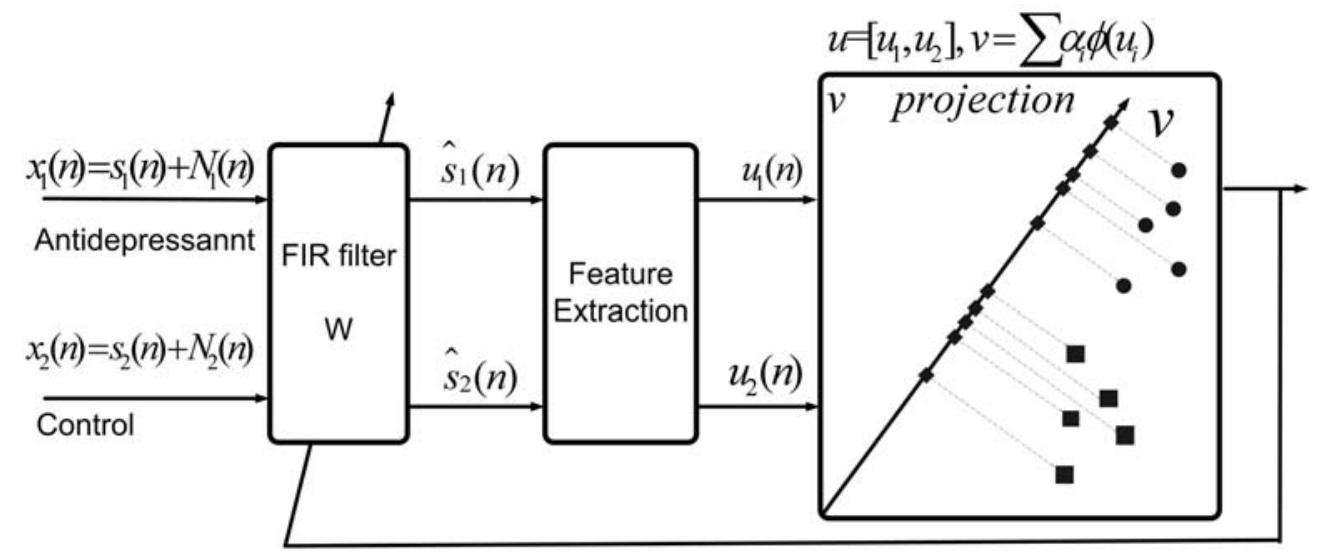

KFD criterion

Fig. 4. Kernel Fisher discriminant based optimal FIR filter tries to find the coefficients of the FIR filter that results in a vector $v$ in feature space, on which projections of points give the maximum separation of the mean scaled in the feature space and the minimum variance in that direction.

in the neighborhood of a global optimum, it is guaranteed that the algorithm converges to the global optimum function value. It works by iteratively dividing the search domain into boxes that have exactly one function value at the box's center. At first, it transforms the search space to be the unit hypercube. The function is then sampled at the center point of this cube. The hypercube is then divided into smaller hyperrectangles whose center-points are also sampled. In each iteration, through evaluation the objective function at those centers, the algorithm determines which boxes are most likely to have a better point than the current optimal one. A box is considered potentially optimal, if it has the potentially best function value for a given Lipschitz constant. The process continues after a prespecified number of function evaluations [17]. A detailed example of the search domain in the DIRECT algorithm was given in [18]. The serial and the parallel implementations of the algorithm have been discussed in [19] and [20], respectively.

\section{Methods}

\section{A. Experimental setup}

The experiments have been performed at the site of an industrial cooperation partner.

1) Forced swimming test procedure: The forced swimming test procedure is as described in [3]. Briefly, each animal is placed to swim for $15 \mathrm{~min}$ in a cylinder (height: $40 \mathrm{~cm}$; diameter: $18 \mathrm{~cm}$ ) containing $18 \mathrm{~cm}$ of water at $25^{\circ} \mathrm{C}$ (pretest session). They are then taken out and allowed to dry for $20 \mathrm{~min}$ in a cage placed below an infrared lamp. Twentyfour hours after the pre-test session, they are again placed in the cylinder for $5 \mathrm{~min}$ (test session), and their behavior is recorded with a camcorder, while it is assured that the camera lens and water line are on a horizontal line in order to minimize the area of distortion due to reflections on the water surface.

2) Calculation of activity profiles: The image analysis software Halcon 7.0 (MVTec Software GmbH, Munich, Germany) was used to analyze the video tapes of rat movements.

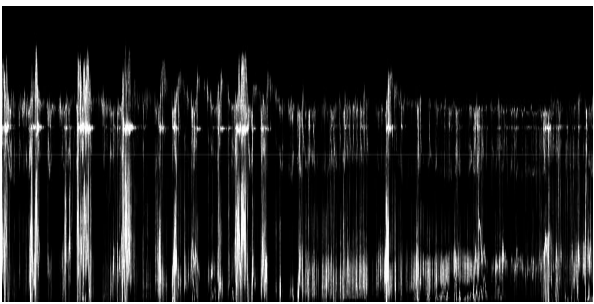

(a) Vehicle-treated

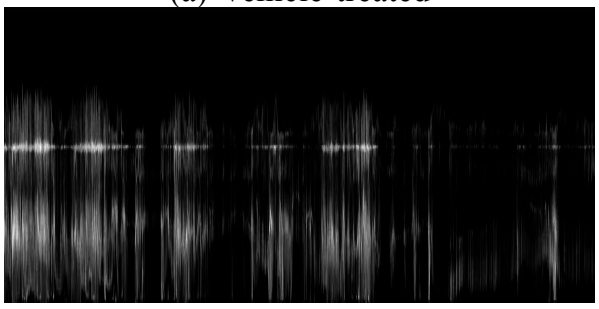

(b) Desipramine-treated

Fig. 5. Activity images of two representative rats treated with a) vehicle $(0.5 \%$ tylose, p.o.) and b) desipramine $(30 \mathrm{mg} / \mathrm{kg}$, p.o.)

To extract the activity profile showing the movement of a rat, for five consecutive frames, the difference between the previous and the next image was calculated and binarized with a fixed threshold and then totalized into one gray level image. Within each activity image all non-zero pixels are summed up in the vertical direction to obtain one activity profile of the whole animal. Fig. 1 shows the corresponding activity profiles of Fig. 5 .

\section{B. Computational setup}

We considered the FIR filter coefficients ( $W$ in Fig. 4 ) in the range of $[-1,1]$ and the length of 10 for that. In order to reduce the dimension for our classification problem, we computed the AR parameters of each activity profile via the recursive solution of the Yule-Walker equation (Levinson method [15]). The optimum value of the order of the AR model, $p_{\text {opt }}$ was gained though Akaike's Information 
Criterion [16]. In our experiment, the average value of 5 was gained for $p_{\text {opt }}$. In the next step, we considered an RBF (Radial Basis Function) kernel for the Kernel fisher discriminant analysis with $\gamma=1$ ([24]). Then, we used the optimization method told above, to find the optimum values of the FIR filter coefficients, $W_{\text {opt }}$. In the next step, we used a SVM classifier with a linear kernel. We found the optimum $C$ parameter of the SVM classifier using a simple grid search in the range of $\left[2^{-2}, 2^{15}\right]$ in terms of the maximum accuracy of the classifier.

\section{RESUlTS AND DISCUSSION}

We used the computational methods described above to classify rats treated with antidepressants of two different classes, tricyclic (imipramine 40mg/kg: 72 rats; desipramine $30 \mathrm{mg} / \mathrm{kg}$ : 112 rats), against a control group treated with vehicle ( $0.5 \%$ tylose: 218 rats).

The prediction quality was then evaluated by specificity (Spec.), sensitivity (Sen.), accuracy (Acc.) and Matthew's correlation coefficient (Mcc.) as follows:

$$
\begin{aligned}
& \text { Sen. }=\frac{T P}{(T P+F N)} \\
& \text { Spec. }=\frac{T N}{(T N+F P)} \\
& \text { Acc. }=\frac{T P+T N}{(T N+F N+T P+F P)} \\
& \text { Mcc. }=\frac{T P \times T N-F N \times F P}{\sqrt{(T N+F N)(T P+F N)(T N+F P)(T P+F P)}}
\end{aligned}
$$

where $T P=$ number of true positives, $T N=$ number of true negatives, $F P=$ number of false positives and $F N=$ number of false negatives,

To show the effect of the FIR filter on the classifier, we first do not use the FIR filter but only an SVM classifier with the RBF kernel. The optimum values of parameters $\gamma$ of the RBF kernel and $C$ in SVM classifier are gained through a grid search method and in terms of maximum average accuracy for the classifier with 5 -fold cross validation. Tables I shows the results of the classifier.

TABLE I

PERFORMANCE OF SVM CLASSIFIER WITH OPTIMUM PARAMETERS OF $\gamma$ AND $C$ (WITHOUT OPTIMUM FIR FILTER) IN CLASSIFICATION ANTIDEPRESSANT DRUGS VS. CONTROL.

\begin{tabular}{lcccc}
\hline Experiment & Spec. (\%) & Sen. (\%) & Acc. (\%) & Mcc. \\
\hline Imipramine 40mg & 79.1 & 85.3 & 80.0 & 0.58 \\
Desipramine 30mg & 75.4 & 85.1 & 76.1 & 0.46 \\
\hline
\end{tabular}

Tables II shows the results of our new method with the FIR filter as described before. As we see, there is a significant improvement in performance with our method.

Fig. 6 shows the activity profiles of tylose and imipramine $40 \mathrm{mg}$ before and after filtering. It is apparent that the data of the filtered activity profiles are more distinguishable than that of the unfiltered activity profiles. In our method, the classifier extracts the features from the filtered activity profiles, while in the second method the SVM classifier works on the
TABLE II

PERFORMANCE OF OUR METHOD (OPTIMUM FIR FILTER) IN CLASSIFICATION ANTIDEPRESSANTS DRUGS VS. CONTROL.

\begin{tabular}{lcccc}
\hline Experiment & Spec. (\%) & Sen. (\%) & Acc. (\%) & Mcc. \\
\hline Imipramine 40mg & 93.1 & 93.3 & 93.3 & 0.84 \\
Desipramine 30mg & 98.0 & 81.7 & 86.6 & 0.75 \\
\hline
\end{tabular}

unfiltered data and tries to find the optimum values of $\gamma$ and $C$ parameters in terms of classification accuracy, and this may lead to overfitting and can result in an increased error rate for new unseen activity profiles. Fig. 7 shows the effect of the FIR filter on the feature space. In Fig. 7.b, in which we have used the FIR filter, the features are more discriminative compared with the features from the unfiltered activity profile which are influenced from the interference noise (Fig. 7.a).

\section{Conclusions}

In this paper, we implemented a new method for classification of biological signals in general, and used it in the animal behavior classification as an example. The hypothesis behind our method is that if we can deduce the interference signal from the original signal, the accuracy of the classifier increases, otherwise, if our features are influenced from that interference signal, the classifier faces overfitting and can not classify them accurately. We used a FIR filter to filter out those additive noise from the signal. The parameters of the FIR filter were obtained via maximizing the accuracy of a classifier that tries to make discrimination between two classes of the activity profiles (e.g. drug vs. control). We used the kernel Fisher discriminant as a criterion for the discrimination and the DIRECT search method for solving the optimization problem. We suggested AR parameters as suitable features for extraction of the dynamic behavior of rats in the forced swimming test. Our proposed behavior classification method allowed for a very reliable discrimination of rats administered to different classes of antidepressant drugs (imipramine and desipramine) versus a vehicle-treated group.

We believe, with some modifications, our proposed FIR based classifiers can also be used in other biological applications such as EEG signals classification and Brain Control Interface (BCI).

\section{REFERENCES}

[1] S. Mika, G. Rätsch, J.Weston, B. Schölkopf, and K.-R. Müller, "Fisher discriminant analysis with kernels," Neural Networks for Signal Processing IX. IEEE, 1999a, pp. 41-48.

[2] M.M. Beigi and A. Zell, "A novel kernel-based method for local pattern extraction in random process signals," 11th European Symposium on Artificial Neural Networks (ESANN), pp. 265-270, 2007.

[3] R.D. Porsolt, G. Anton, N. Blavet, M. Jalfre, "Behavioural despair in rats: A new model sensitive to antidepressant treatments," Eur. J. Pharmacol, vol. 47, pp. 379-391, 1978.

[4] F. Borsini, and A Melt, "Is the forced swimming test a suitable model for revealing antidepressant activity?," J. Psychopharmacology, vol. 94, pp. 147-160, 1988.

[5] J. Cryan and I. Lucki, "Antidepressant-like effects mediated by 5hydroxytryptamine2c receptors," J. Pharmacol. Exp. Ther, vol. 295, pp. 1120-1126, 2000. 

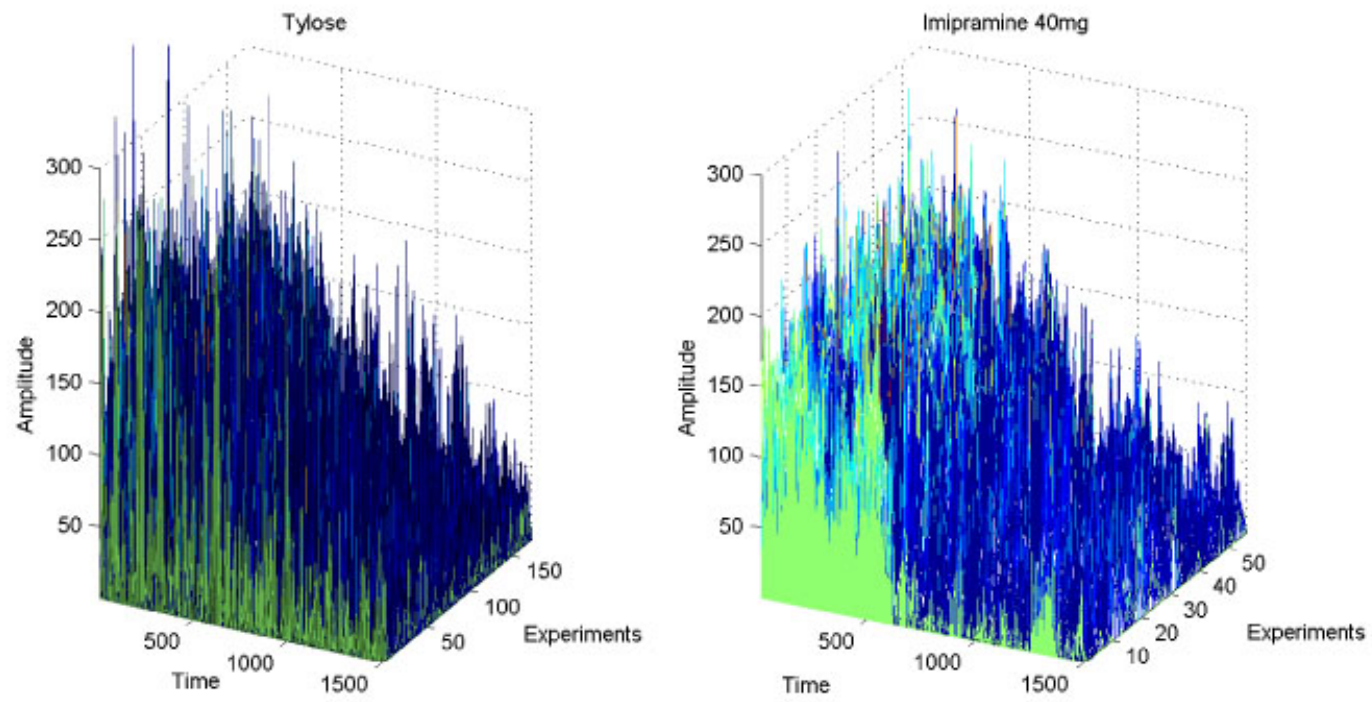

(a) Activity profiles
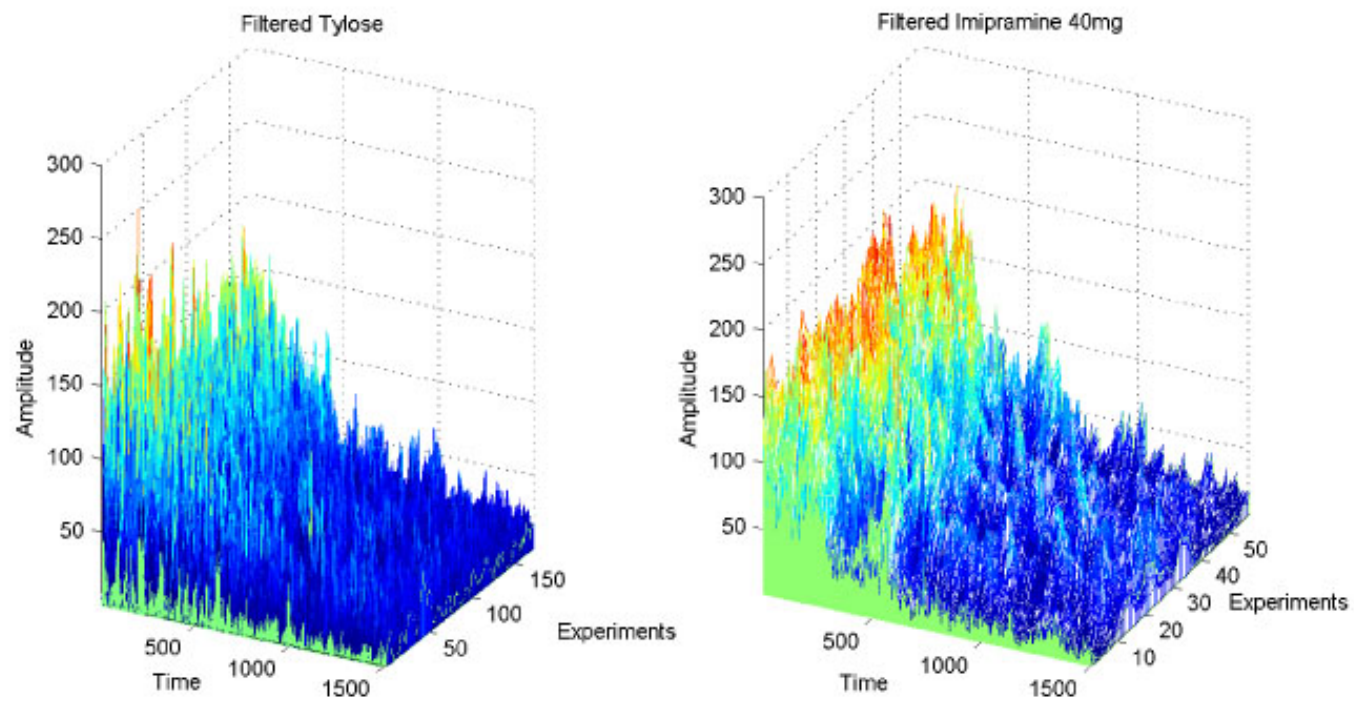

(b) Activity profiles after the obtained FIR filter

Fig. 6. The effect of the obtained optimal FIR filter on the activity profiles of tylose and imipramine 40mg. a)Raw data of activity profiles. b) Filtered activity profiles.

[6] J. Cryan, A. Markou, and I. Lucki, "Assessing antidepressant activity in rodents: recent developments and future needs," Trends. Pharmacol. Sci., vol. 23, pp. 238-245, 2002.

[7] M. Detke, M. Rickels, and I. Lucki, "Active behaviors in the rat forced swimming test differently produced by serotonergic and noradrenergic antidepressant," Psychopharmacology., vol. 121, pp. 66-72, 1995.

[8] S-J Kim, A. Magnani, and S. Boyd, "Optimal Kernel Selection in Kernel Fisher Dicriminant Analysis," Proceedings of the 23rd International Conference on Machine Learning (ICML 2006). 2006, pp. 465-472.

[9] F. Lotte, M. Congedo, A. Lecuyer, F. Lamarche, and B. Arnaldi, "A review of classification algorithms for EEG-based brain-computer interfaces," J Neural Eng.. vol. 4, pp. R1-R13, 2007.

[10] V.P.S Naidu, and M.R.S Reddy, "Autoregressive (AR) based power spectral analysis of heart rate time series signal (HRTS signal)," TENCON 2003. Conference on Convergent Technologies for Asia-Pacific Region. vol. 4, pp. 1391 - 1394, 2003.

[11] C. W. Anderson, E. A. Stolz and S. Shamsunder, "Multivariate Autoregressive Models for Classification of Spontaneous Electroen- cephalogram During Mental Tasks", IEEE Trans Biomed Eng.. vol. 45(3), pp. $277-286,1998$.

[12] N-J Huan, and R. Palaniappan, "Neural network classification of autoregressive features from electroencephalogram signals for braincomputer interface design," J. Neural Eng.. vol. 1(3), pp. 142 - 50, 2004.

[13] G. U. Yule, "On a method of investigating prediction in disturbed seris. With special reference to Wölfer's sunspot numbers," Philos. Trans. of Royal Soc. series A.. pp. 226 - 267,1927

[14] G. Walker, "On periodicity in series of related terms", Proceedings of the R. Soc. of London. Series A.. vol. 131, pp. 518 - 532,1931

[15] N. Levinson, "The Wiener RMS (root-means-squares) error criterion in filter design and prediction," J. Math Phys. vol. 25, pp. $261-278,1947$

[16] H. Akaike, "A new look at the statistical model identification", IEEE Trans. Automat. contr. vol. 22, pp. 203-217, 1974.

[17] D. R. Jones, C. D. Perttunen, and B. E. Stuckman, "Lipschitzian optimization without the Lipschitz constant", Journal of Optimization Theory and Applications, vol. 79, pp. 157-181, 1993 

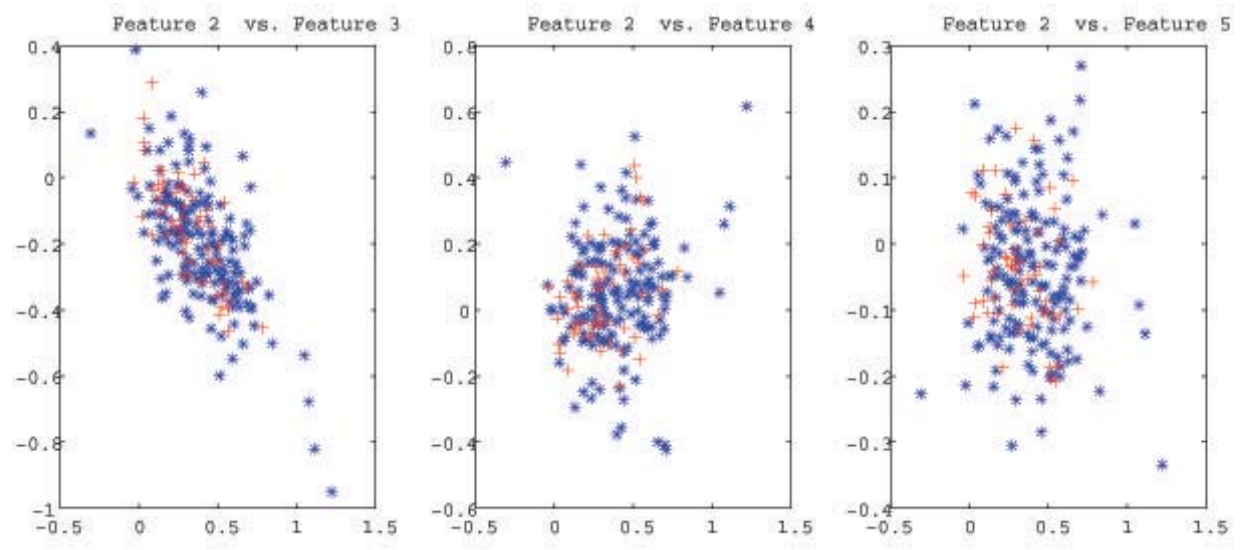

(a) AR features of an instance of activity profile
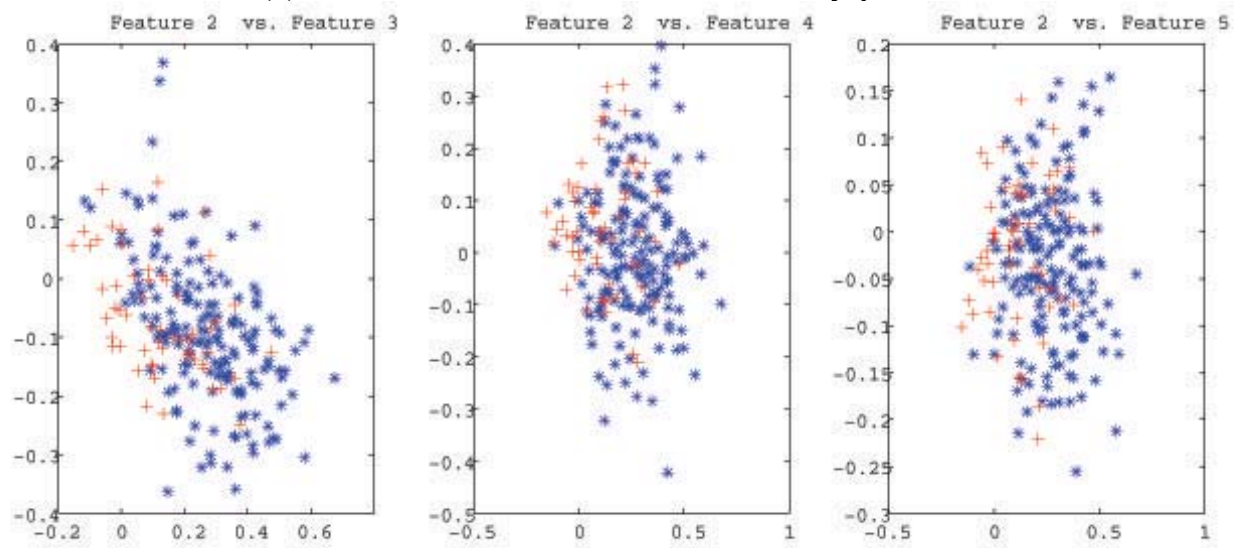

(b) AR features of the activity profile after FIR filter

Fig. 7. The effect of the obtained optimal FIR filter on the AR features of an instance of activity profile of tylose (blue *) and that of imipramine $40 \mathrm{mg}$. $($ red + ). a) without FIR filter. b) with FIR filter.

[18] L. T. Watson and C. A. Baker, "A fully-distributed parallel global search algorithm", Engineering Computations, vol. 18, no. 1/2, pp. 514$579,2001$.

[19] J. He, L. T. Watson, N. Ramakrishnan,C. A. Shaffer, A. Verstak, J. Jiang, K. Bae, and W. H. Tranter, "Dynamic data structures for a direct search algorithm," Computational Optimization and Applications, vol. 23, pp. 5-21, 2002.

[20] J. He, M. Sosokina, L. T. Watson, and J. W. Zwolak, "Data-distributed parallelism with dynamic task allocation for a global search algorithm", Proc. High Performance Computing Symposium, M. Parashar and L. Watson (eds.), Society for Modeling and Simulation International, San Diego, CA, pp. 164-172. 2005.

[21] P. Moser, and D. Sanger, "5-HT1A receptor antagonist neither potentiate nor inhibit the effects of fluoxetine and befloxatone in forced swim test in rats," Eur. J. Pharmacol., vol. 372, no. 12, pp. 127-134. 1999.

[22] K. Takamori, S. Yoshida, and S. Okuyama, "Effect of ACTH on the imipramine and desipramine-induced decrease in duration of immobility time as measured in a rat forced swimming test", J. Life Sciences, vol. 69, pp. 1891-1896. 2001.

[23] I. Belozertseva, T. Kos, P. Popik, W. Danysz, and A. Bespalov, "Antidepressant-like effects of mGluR1 and mGluR5 antagonists in the rat forced swim and the mouse tail suspension tests," J. Eur. Neuropsychopharmacol., Epub ahead of print. 2006.

[24] C-C Chang and C-J Lin, "LIBSVM: a library for support vector machines," Software available at http://www. csie.ntu.edu. tw/ Cjlin/libsvm, 2001.

[25] H. Fröhlich, G. Birk, H. Rosenbrock, and A. Zell, "Automated Classification of the Behavior of Rats in the Forced Swimming Test with Support Vector Machines," Journal of Neural Networks (Elsevier). to appear. 